\title{
Hypokalaemia-induced rhabdomyolysis as the presenting feature of primary hyperaldosteronism
}

\author{
Karunaratna WGSG ${ }^{1}$, Dilhani PDW ${ }^{1}$, Edirisinghe LU $^{1}$, Sumanatilleke $\mathbf{M}^{1}$, Weerarathna $\mathbf{T P}^{2}$, \\ Bodinayake $\mathbf{C K}^{2}$ \\ ${ }^{\prime}$ Teaching Hospital Karapitiya, Galle, Sri Lanka \\ ${ }^{2}$ Department of Medicine, Faculty of Medicine, University of Ruhuna, Galle, Sri Lanka
}

Correspondence: Dr. Shashi Karunaratna;

Mobile:+94777007551; e-mail: shashikaru@yahoo.com

\section{Introduction}

Primary hyperaldosteronism (PHA) is a well established cause of secondary hypertension. It is characterised by hypertension, hypokalaemia and metabolic alkalosis. In some extreme conditions, PHA can induce excessive potassium excretion resulting in profound hypokalaemia followed by rhabdomyolysis. Although muscle weakness is a well known manifestation of marked hypokalaemia, rhabdomyolysis is a rare presentation of hypokalaemia (1). Rhabdomyolysis presenting with severe hypokalaemia as the first manifestation of primary hyperaldosteronism is extremely rare (2). We report a 38-year-old female with arterial hypertension who presented to the Emergency Department with generalised muscle weakness associated with rhabdomyolysis as the presenting symptom of PHA.

\section{Case report}

A 38-year-old female with a history of hypertension for 8 years presented with acute onset rapidly progressive muscle weakness mainly affecting proximal girdle muscles for four days. On physical examination she had a lean body habitus with a BMI of $18 \mathrm{~kg} / \mathrm{m}^{2}$. She also had a multinodular goitre but was euthyroid, clinically. Her blood pressure was 170/90 $\mathrm{mmHg}$, both supine and standing positions. Nervous system examination of both upper limbs and lower limbs revealed marked proximal muscle weakness. Deep tendon reflexes were normal while plantars were flexor, bilaterally. There was no sensory deficit.

Investigations revealed normal basic haematological parameters, severe hypokalaemia, increased renal potassium loss and high creatine phosphokinase (CPK) level (Table). Based on these findings we established the diagnosis of rhabdomyolysis due to hypokalaemia. Intravenous potassium supplementation was initiated. Proximal muscle weakness completely recovered after correction of hypokalaemia and serum CPK levels normalized within one week.

Further investigations revealed elevated plasma aldosterone / renin ratio and CT scan of the abdomen revealed bilateral adrenal hyperplasia with a dominant nodule in the left side (Figure). Adrenal vein sampling was not done due to financial constraints.

The diagnosis of PHA due to bilateral adrenal hyperplasia was confirmed and she was started on spironolactone. Potassium supplements were gradually withdrawn. Blood pressure and serum potassium level remained normal and clinically there was no proximal muscle weakness during five months of follow up.

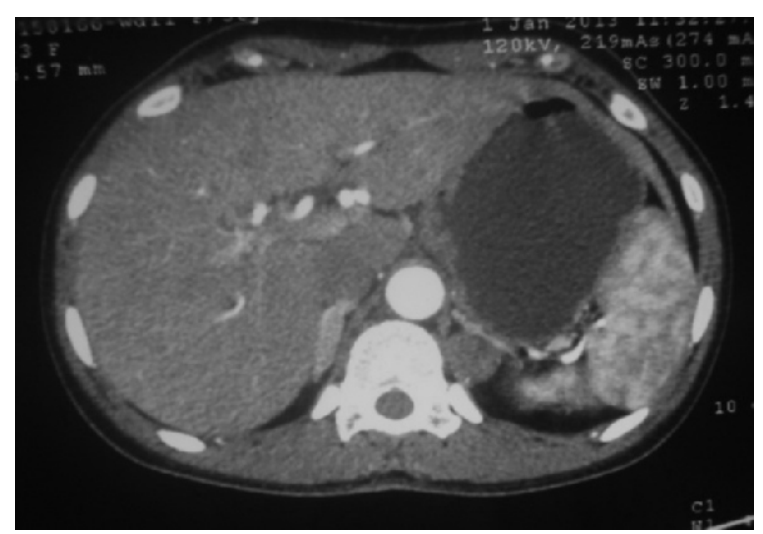

Figure: Contrast enhanced CT scan of the abdomen showing bilateral adrenal hyperplasia with a dominant nodule on the left side. 


\section{Table: Investigation results}

Serum electrolytes: $\quad \mathrm{Na}-148 \mathrm{mmol} / \mathrm{L}, \mathrm{K}-1.9 \mathrm{mmol} / \mathrm{L}$;

Spot urine electrolytes: Na $10 \mathrm{mmol} / \mathrm{L}, \mathrm{K} 25 \mathrm{mmol} / \mathrm{L}$

24 hour urinary excretion of electrolytes: $\mathrm{Na}-55 \mathrm{mmol} / \mathrm{L}, \mathrm{K}-38 \mathrm{mmol} / \mathrm{L}$

Creatine Phosphokinase: 2840 U/L (26 -192); Repeat test: 2499 U/L

Thyroid function tests

- TSH: $0.546 \mu \mathrm{IU} / \mathrm{mL}(0.4-4.0)$

- Free T4: $1.43 \mathrm{ng} / \mathrm{dL}(0.89-1.76)$

- Free T3: 4.66pmol/L (4- 8.3)

Antithyroid peroxidase (microsomal) antibodies (TPO): $3.5 \mathrm{IU} / \mathrm{mL}$

ESR: $20 \mathrm{~mm} / 1^{\mathrm{st}} \mathrm{hr}$

Arterial Blood Gas analysis

- $\mathrm{pH} \quad-7.638(7.35-7.45)$

- $\mathrm{pCO}_{2}-29.2 \mathrm{mmHg}(35-46)$

- $\mathrm{pO}_{2}-85.9 \mathrm{mmHg}(75-100)$

- $\mathrm{HCO}_{3}-31.6 \mathrm{mmol} / \mathrm{L}(22-26)$

Plasma renin activity (PRA): $0.7 \mathrm{ng} / \mathrm{ml} / \mathrm{hr}(0.1-3.1)$

Aldosterone: $23.6 \mathrm{ng} / \mathrm{dl}$ (supine $30 \mathrm{~min}$ ) (3 to $16 \mathrm{ng} / \mathrm{dl}$ )

Plasma Aldosterone/ renin ratio: $33.7(<20)$

Overnight dexamethasone suppression test: $41.5 \mathrm{nmol} / \mathrm{L}(<50 \mathrm{nmol} / \mathrm{l})$

Serum calcium: $2.4 \mathrm{mmol} / 1(2.1-2.5)$

Serum Mg: $1.6 \mathrm{mg} / \mathrm{dl}(1.6-2.6)$

Blood urea: $34 \mathrm{mg} / \mathrm{dL}$

Serum creatinine: $98 \mu \mathrm{mol} / \mathrm{L}$

24 Hours Urinary VMA excretion: $5.1 \mathrm{mg} / 24 \mathrm{hrs}(1-11)$

USS abdomen: No suprarenal masses seen

\section{Discussion}

PHA is characterised by hypertension, hypokalemia, suppressed PRA, and increased aldosterone excretion (3). Bilateral adrenal hyperplasia and aldosterone producing adenoma are the most common subtypes of PHA(1).

Rhabdomyolysis is defined as a pathological condition of skeletal muscle cell damage leading to the release of toxic intracellular material into the blood circulation, such as creatine phosphokinase (2). The major causes of rhabdomyolysis include trauma, excessive muscle activity, and hereditary muscle enzyme defects, and a variety of medical causes, including drugs and toxins, muscle hypoxia, metabolic and endocrine disorders, infections, and temperature alterations. Hypokalaemia is a recognised cause of rhabdomyolysis. When serum concentration falls below $3 \mathrm{mEq} / \mathrm{L}$, malaise, muscular weakness, fatigability may occur. However, muscle enzyme elevation is usually not seen until potassium concentration falls below 2.5 $\mathrm{mEq} / \mathrm{L}$. Frank rhabdomyolysis is usually observed only when serum potassium values are below 2 $\mathrm{mEq} / \mathrm{L}(4)$.

Rhabdomyolysis is not common in PHA although hypokalaemia induced myopathy is a well known association. In this case, rhabdomyolysis was the presenting symptom of PHA. Correction of hypokalaemia with intravenous potassium 
supplements resolved rhabdomyolysis normalising creatine phosphokinase values, before hyper-aldosteronism was treated with spironolactone. This supports that the main cause of rhabdomyolysis was hypokalemia and not the direct effect of aldosterone (4).

In conclusion, hypokalaemia severe enough to cause rhabdomyolysis can be a rare presenting manifestation of PHA. Also when rhabdomyolysis, hypokalemia and metabolic alkalosis occur together in a hypertensive patient, PHA should be considered and further investigation for PHA should be initiated (4).

\section{References}

1. Karagüzel G, Bahat E, Imamoglu M, Ahmetoglu A, Yildiz $\mathrm{K}$, Okten A. An unusual case of an aldosterone-producing adrenocortical adenoma presenting with rhabdomyolysis. Journal of Paediatric Endocrinology and Metabolism 2009; 22(11): 1087-90.
2. Zhang Wen, Li Chuanwei, Zeng Chunyu, Huang Hui and Li Weimin. Rhabdomyolysis presenting with severe hypokalaemia in hypertensive patients: a case series BMC Res Notes. 2013 Apr 17; 6: 155.

3. Panagiotis Kotsaftis, Christos Savopoulos, Dimitrios Agapakis, George Ntaios, Valentini Tzioufa, Vasilios Papadopoulos, Epaminondas Fahantidis, Apostolos Hatzitolios. Hypokalaemia induced myopathy as first manifestation of primary hyperaldosteronism an elderly patient with unilateral adrenal hyperplasia: a case report. Cases Journal 2009; 2: 6813.

4. Martínez JJ, Oliveira CL, Meneses AL, Rodríguez SA, Corrales PP, López AH, Romero FB. Rhabdomyolysis due to primary hyperaldosteronism. Endocrinol Nutr 2009; 56(8): 431-4. 